\section{§1. Introduction.}

Does there exist a function harmonic or analytic and satisfying a prescribed condition on a given Riemann surface? This question is a principal leading 1 dea in the recent function-theory, especially in the theory of classification, and it leads us to the various deep and important results as well as new notions. Moreover it brings to the various extremal problems to be solved. In this tendency several authors above all Finnish colleagues have contributed to the theory. Although this theory is gained in a great success, there remein many important and unsolved problems.

In the present paper we shall explain a method to classify the Riemann surface. It is directed by the following leading 1dea: Under what conditions does there exist a solution of a partial differential equation of elifptic type $\Delta u=P u$ on a given Riemann surface? Although we have succeeded to establish a classification theory to a certain extent, there remain many problems unsolved, and we have found yet no application to the function-theory.

We first give a more precise explanation on our Riemann surface and differential equation to be considered.

Riemann surface $F$ means here the one in the sense of Weyl-Rado. Its Ideal boundary is denoted by $\Gamma$, and we restrict ourselves to the Riemann surface of intinite genus save when the contrary is explicitly mentioned.

Differential equation considered here is the following type:

(A) $\frac{\partial^{2}}{\partial x^{2}} u(x, y)+\frac{\partial^{2}}{\partial y^{2}} u(x, y)=P(x, y) u(x, y)$

where $z=x+i y$ is a local uniformizing parameter at a point $p$ on $F, P(x, y)$ is a real holomorphic function of $(x, y)$ being positive except at a countable set of zero-points with accumulation points lying only on the 1 deal boundary $\Gamma$. We assume moreover that if we change the local parameter $z$ to $z^{\prime}$, then

$P(z)$ changes as follows:

$$
P(z)=P\left(z^{\prime}\right)\left|\frac{d z^{\prime}}{d z}\right|^{2} \text {. }
$$

For this type of differential equation (A) we can prove the existence of the so-called kernel function and Green functions under various boundary conditions, for instance, the Green function with ordinary, i. $\theta$. vanishing boundary values or the Neumann function, 1.e. the one with vanishing normal derivative along the boundary, and also the solvability of the first boundary value problem. Moreover the maximum or minimum principle and Harnack's convergence theorem are valid with a slight modification. These tacts will bo shortly explained but the precise prool's will be omitted of $f^{2}$. For the precise rormulations one can refer to the S.Bergman's book [1] and S.Eergman-M.Schiffer [1,2]。 For other ways of formulations $c r$. R.Courant-D.HIlbert [1], E.PI card $[I, 2], L . L j$ chtenstein $[i, 2], R$ 。 Nevaniinna [1] and D.Hilbert [1].

\section{$\S 2$. Det'initions and General} Considerations.

Let $\left\{F_{n}\right\} n=0,1,2, \ldots$ be an exhaustion of $F$ in the ordinary sense, that is,

1) $F_{n}$ is a compact connected analytic subregion of $F$, that is, $F_{n}$ is connected and the closure of $F_{n}$, $\bar{F}_{n}$ say, is compact and its boundary consists or a finite number of simple closed analytic curves $\Gamma_{n}^{(v)}(v=1, \cdot, m), \Gamma_{n}=\sum_{v=1}^{m} \Gamma_{n}^{(v)}$.

Especially $F_{0}$ is supposed to be a simply-ccnnected compact analytic subregion of $F$;

$$
\text { 11) } \begin{aligned}
& \bar{F}_{n} \subset F_{n+1} \quad(n=0,1, \cdots) \text {; } \\
& \lim _{n \rightarrow \infty} F_{n}=F .
\end{aligned}
$$

Delinition 2.1. Dirichlet integral $D(u)$ and mixed Dirichlet integral $D(u, v):$ 


$$
\begin{aligned}
& D_{F}(u, v)=\iint_{F}\left(\frac{\partial u}{\partial x} \frac{\partial v}{\partial x}+\frac{\partial u}{\partial y} \frac{\partial v}{\partial y}+P u v\right) d x d y, \\
& D_{F}(u, u)=D_{F}(u) .
\end{aligned}
$$

By the Green's formula, we see that if $\Delta u=P u$, then $D_{F}(u, v)=\int_{\Gamma} v \frac{\partial u}{\partial v} d s$ for any $v$, where $\partial / \partial v$ denotes the dif'ierentiation in the direction $\mathrm{ot}^{\prime}$ outer normal.

Definition 2.2. If $u$ is a solution of $(A)$ and satisfy $D_{F_{n}}(u)<\infty$, then we call $u \in L^{2}\left(F_{n}\right)^{F_{n}}$.

obviously $L^{2}\left(F_{n}\right)$ forms a real Hilbert space with respect to the inner product

$$
(u, v) \equiv D_{F_{n}}(u, v), \quad u, v \in L^{2}\left(F_{n}\right) .
$$

Definition 2.3. Kernel function $K_{n}\left(z, z_{0}\right)$ of $L^{2}\left(F_{n}\right)$ : If $K_{n}\left(z, z_{0}\right)$ $\epsilon I^{2}\left(F_{n}\right)$ has the reproducing property for any $L^{2}\left(F_{n}\right)$-integrable solution $u$ of $(A)$ :

$$
u\left(z_{0}\right)=D_{F_{n}}\left(u(z), K_{n}\left(z, z_{0}\right)\right),
$$

then we call $K_{n}\left(z, z_{0}\right)$ the reproducing kernel function of $L^{2}\left(F_{n}\right)$.

We explain here the existence of $K_{n}\left(z, z_{0}\right)$ - According to N. Aronszajn [I], in a locally uniformly bounded Hilbert space, there exists one and only one reproducing kernel function $K_{n}\left(x, x_{0}\right)$ of $L^{2}\left(F_{*}\right)$, and hence we have only to prove the locally uniformly boundedness. Since Riemann surface is of locally Euclidean character, we may discuss the problem in a Euclidean small disc. Let $u(z)$ be an arbitrary solution of (A) with $D_{F_{n}}(u)<M$, then $\Delta\left(u^{2}\right) \geq 0$. In fact, we have

$$
\begin{aligned}
\Delta\left(u^{2}\right) & =2 u \Delta u+2\left(\frac{\partial u}{\partial x}\right)^{2}+2\left(\frac{\partial u}{\partial y}\right)^{2} \\
& =2\left[P u^{2}+\left(\frac{\partial u}{\partial x}\right)^{2}+\left(\frac{\partial u}{\partial y}\right)^{2}\right] .
\end{aligned}
$$

Let $B_{R, \varepsilon}$ denote a ring domain bounded by $C_{R}\left(\left|z-z_{0}\right|=R\right)$ and $C_{\varepsilon}$ $\left(\left|z-z_{\bullet}\right|=\varepsilon, \varepsilon<R\right)$, we have

$$
\begin{aligned}
& \int_{C_{R}} u^{2} \frac{\partial}{\partial v} \log \left(\frac{R}{\left|z-z_{0}\right|}\right) d s+\int_{C_{z}} u^{2} \frac{\partial}{\partial v} \log \left(\frac{R}{\left|z-z_{0}\right|}\right) d s \\
& =-\iint_{B_{R, \varepsilon}} \log \frac{R}{\left|z-z_{0}\right|} \Delta\left(u^{2}\right) d x d y \\
& \leqq 0 \text {. }
\end{aligned}
$$

Letting $\mathcal{E}$ tend to zero, we have

$$
2 \pi R u^{2}\left(z_{0}\right) \leqq \int_{0}^{2 \pi} u^{2} R d \theta,
$$

and integrating with respect to $R$ Irom $r$ to $R, 0<r<R$, we have

$$
\begin{aligned}
\pi u^{2}\left(x_{0}\right) & \left(R^{2}-r^{2}\right) \leqq \iint_{B_{R, \varepsilon}} u^{2} d x d y \\
& \leqq \frac{1}{P_{0}} \int_{B_{R, \varepsilon}} P u^{2} d x d y \leqq \frac{1}{P_{0}} D_{R}(u) \\
& <\frac{1}{P_{0}} \cdot M,
\end{aligned}
$$

where $P_{0}=\inf _{r<\left|z-z_{0}\right|<R} P(z)$ and $P_{0}>0$

in view of the isolatedness of the zero-points of $P(x)$. Thus $u(x)$ is locally uniformly bounded, what leads to the existence of $K_{n}\left(z, z_{0}\right)$.

Definition 2.4. Green function of (A). Green function $g_{n}\left(x, z_{0}\right)$ (the ordinary one) is a fundamental solution of (A) in $F_{n}$ and $g_{n}\left(x, x_{0}\right)$ $\equiv 0$ on $\Gamma_{n}$, that is, $\Delta g_{n}=P g_{n}$ for $x\left(\neq z_{0}\right) \in F_{*}$ and $g_{n}\left(z, z_{0}\right)$ $-A\left(z, x_{0}\right) \log \left(1 / 1 z-z_{d}\right)$ is a holomorphic solution $J I^{\circ}(A)$ where $A\left(z, z_{0}\right)$ is also a holomorphic tunction satisfying $\lim _{z \rightarrow x_{0}} A\left(x, z_{0}\right)=1$, and moreover $g_{n}=0$ on $\Gamma_{n}$.

Once the existence of the kernel function and a fundamental solution having been established, we obtain the existence and the expression of Green function in the following way: Let $S\left(z, x_{0}\right)$ and $K_{n}\left(z, x_{0}\right)$ be a fundamental solution and the kernel function in $F_{n}$, respectively. If we put $\sigma\left(z_{0}, w\right)=D_{F_{n}}\left(K_{n}\left(z_{1} z_{0}\right), S\left(z_{0}, w\right)\right)$, then we see that

$$
S(z, w)-\sigma(z, w)
$$

is the desired Green function, a fact following from the consideration of uniqueness of $g_{n}$ which is easily proved from the assumption. For the existence of a fundamental solution of (A) one can refer to the Hilbert's book [1] and F.Holmgren [1].

obviously we have an inequality

$$
\left|\frac{\partial}{\partial v} g_{n}\left(z, x_{0}\right)-\frac{\partial}{\partial v} g_{n}\left(z_{1}, z_{0}\right)\right| \leqq 11\left|z-z_{1}\right| \text {, }
$$

and, by means of the Green's formula, the Poisson integral representation

$$
u\left(z_{0}\right)=-\int_{\Gamma_{n}} u(x) \frac{\partial g_{n}\left(x, x_{0}\right)}{\partial v} d s,
$$

where $u\left(z_{0}\right) \in L^{2}\left(F_{n}\right)$. Thus we can conclude that the so-called Harnack's convergence theorem remains valid in a slight modified form in our case. 
Definition 2.5. Neumann function of (A). Noumann function $N_{n}\left(x, x_{0}\right)$ is a lundamental solution of (A) in

$$
F_{n} \text { and } a N_{n}\left(x, x_{0}\right) / \partial \nu \geq 0 \text { on } \Gamma_{n} \text {. }
$$

The existence of $N_{n}\left(z, x_{0}\right)$ is easily proved. Furthermore, its explicit expression is given by

$$
N_{n}\left(z, z_{0}\right)=2 \pi K_{n}\left(z, z_{0}\right)+g_{n}\left(z, z_{0}\right) .
$$

For our kernel iunction $K_{x}\left(z, z_{0}\right)$, we have

$$
\text { j.) } K_{n}\left(z, z_{0}\right)=K_{n}\left(x_{0}, z\right) \text { and } K_{n}\left(x_{0}, z_{0}\right) \geq 0 \text {; }
$$

$$
\text { 11) If } x \leq m \text {, then }
$$$$
K_{n}\left(z_{0}, z_{0}\right) \geq K_{m}\left(z_{0}, z_{0}\right), z_{0} \in F_{n} ;
$$

1i1) $I f\left\{g_{v}(x)\right\} \quad, \nu=1,2, \ldots$

is a complete orthonormal system in

$$
\begin{aligned}
K^{z}\left(F_{n}\right) & , \text { then } \\
K_{n}\left(x, z_{0}\right) & =\sum_{\nu=1}^{\infty} g_{\nu}(z) g_{\nu}\left(z_{0}\right) .
\end{aligned}
$$

These facts are obtalned in more abstract way in the Hilbert space with a reproducing kemel function; cr. N.Aronszajn [1]. Especially In our case we have

$$
\text { 1v) } K_{n}\left(z, z_{0}\right) \geq 0 \text {. }
$$

This is proved by S.Bergman-M.Schiffer [I].

Here we shall explain two lemata ol which we shall often make use in later.

Lemma 2.1. If $u$ and $v$ are two solutions of $(A)$ and $u \geq v$ on $\Gamma n$, then $u \geqq v$ on $F_{n}$.

Proot. Iet $\psi=u-v$, then $\Delta \psi=P \Psi$. Let $D$ be a pointset satisfying $\psi<0$, then a connected component $D_{1}$ of $D$ is a subregion of $F_{n}$ and $\Gamma_{n} \cap D=0$. Now $\psi$ is superharmonic on $D_{1}$, and hence $0>\psi(x) \geq \inf \psi(x)=\operatorname{im}_{(0)} \psi^{\prime}(x)=0$, where $\left(D_{1}\right)$ is the boundary $D_{1} D_{1}$. Thus $D_{1}$ and hence $D$ also is an empty set. Therefore $\psi(z) \geq 0$ on $F_{n} \cdot q \cdot \theta \cdot d$.

Lemma 2.2. If $u$ and $v$ satisfy the equation $\Delta u=0$ and $\Delta v=P v$, respectively, and $u \geq v \geq 0$ on

$\Gamma_{n}$, then $u \geq v$ on $F_{n}$.

Proof. By Lemma $\alpha . I$, we have $v \geqq 0$ on $F_{n}$, and hence if we put $\boldsymbol{\psi}=\boldsymbol{u}-\boldsymbol{v}$, then $\psi$ is superharmonic on $F_{n}$ and $\geqq 0$ on $\Gamma_{n}$. Therefoie we have $\psi \geq 0$ an' $F_{n}$ and hence $u \geq v$ on $F$.
To solve the first boundary value problem, wa can also make use of the so-called Schwarz's alternating process and the method of successive approximation used by E.F1card $[1,2]$. or more directly we can prove the solvability and expression named polssor integral formula by making use of the kernel function or Green function. And the uniqueness of the solution can be deduced by Lemma 2.1 .

The following Lerma is a precision of Lemma $2 . \dot{2}$.

Lemma 2.3. Let $u$ be a solution of $(A)$ on $F_{n}$ satisfying $u=1$ on $\Gamma_{n}$, then $0 \leqq u \leqslant 1$ in $F_{n}$; the right hand inequality being of the strict sense, that is, the equality sign being excluded.

Proof. By Lemma $2.2,0 \leqq u \leqq 1$ is obtained. If $u\left(z_{0}\right)=1$ for a point $z_{0} \in F_{n}, \notin \Gamma_{n}$, then $\frac{\partial u}{\partial x_{0}}=\frac{\partial u}{\partial y_{0}}=0$ and $\Delta u\left(z_{0}\right) \leqq 0$

On the other hand $\Delta u\left(x_{0}\right)=P\left(x_{0}\right) u\left(x_{0}\right) \geqq 0$, and thus we have $\mathrm{P}\left(x_{0}\right)=0$. Hence $u(z) \lessgtr 1$ for any point $z \in F_{n}$ such that $P(x)>0$. By the isolatedness of the zero-points of $P(z)$, we have $P(z) ¥ 0$ for $0<\left|z-z_{0}\right| \leq \varepsilon$, where $\varepsilon$ is a sufficientiy small positive number. Thus, for such a point $z$ $u(z) \& i$. On the other hand, the subharmonicity of $u(z)$ implies that

$$
u\left(z_{0}\right) \leqq \frac{1}{2 \pi \varepsilon} \int_{0}^{2 \pi} u(z) \varepsilon d \theta .
$$

Hence $L=u\left(z_{0}\right) \lessgtr 1$, which is absurd. Thus we have the desired result.

Definition 2.6. Let $\omega_{n}\left(x, I_{n}\right.$, $\left.F_{n}-F_{0}\right)$ be the inite solution of (A) in $\boldsymbol{F}_{n}-F_{0}$, being identicaliy $I$ on $\Gamma$ and $O$ on $\Gamma$.

Detinition 2.7. Let $\Omega_{n}\left(x, \Gamma_{n}, F_{n}\right)$ be the inite solution of (A) in $F_{n}$, and be identically $I$ on $\Gamma_{n}$.

By Lemma 2.1, we havo, for $m \geqq n$,

$1 \geqq \omega_{n}\left(x, \Gamma_{n}, F_{n}-F_{0}\right) \geqq \omega_{m}\left(x, \Gamma_{n}, F_{m}-F_{0}\right)$ 30 ,

$$
z \in F_{n}-F_{0} \text {, }
$$

and hence the Iimit

$$
\lim _{n \rightarrow \infty} \omega_{n}\left(x, \Gamma_{n}, F_{n}-F_{0}\right)=\omega\left(x, \Gamma, F-F_{0}\right)
$$


exists. Similarly, there exists

$$
\lim _{n \rightarrow \infty} \Omega_{n}\left(z, \Gamma_{n}, F_{n}\right)=\Omega(z, \Gamma, F) \text {. }
$$

Detinition 2.8. F $\in 0_{\omega}$ means that $\omega\left(2, \Gamma, F-F_{0}\right) \equiv 0$

Definition 2.9. $F \in O_{\Omega}$ means that $\Omega(x, \Gamma, F)=0$.

That these classes are determined independ of a special choise of $F$. and the process of exhaustion, can be proved analogously to the harmonic case.

Definition 2.10. If there is no non-constant bounded solution of (A) on $F$, then we call $F \in O_{B}$.

Definition 2.11. If there is no non-constant solution of $(A)$ on $F$, having finite Dirichlet integral $D_{F}(u)$, then we write $F \in O_{D}$.

From Lemma 2.1 , we have for $m \geq n$,

$$
0 \leqq g_{n}\left(z, z_{0}\right) \leqq g_{m}\left(z, z_{0}\right), \quad z \in F_{n},
$$

and hence the limiting function

$$
\begin{aligned}
& \lim _{n \rightarrow \infty} g_{n}\left(z, z_{0}\right)=g\left(z, z_{0}\right) \text {. } \\
& \text { Delinition 2.12. (a) If there is } \\
& \text { a point } z \in z_{0}, z_{0} \text {, such }
\end{aligned}
$$
that $g\left(z, z_{0}\right)=\infty$ for a fixed point $z_{0} \in F$, then we write

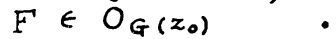

(b) $F \in O_{G}$ means that there is at least one point $z_{0}$, such that $F \in O_{G\left(z_{0}\right) ; n a m e l y, F} ; O_{G}$ means that there is no point $z_{0}$, such that $F \in O_{G\left(z_{0}\right) \text {. }}$

$$
\text { Since } K_{n}\left(z_{0}, z_{0}\right) \text { is non-nega- }
$$

tive ana decreases as $n$ ircreases, the IImit $\lim _{n \rightarrow \infty} K_{n}\left(z_{0}, z_{0}\right)=K\left(z_{0}, z_{0}\right) \geqq 0$ exists.

Definition 2.13. $E \in O_{K}$ means that $K(z, z) \equiv 0$ for any point

$$
z \in F \text {. }
$$

It is to be noticed that there is no constant solution of (A) except the vanishing one.

we shall often make use of also tne quantities and definitions with respect to the harmonic case. To avola the coniusion we shall distinguish the harmonic case by the upper index ( $h$ ), for example, harmonic measure is denoted by $\omega^{(h)}$, Green function of $\Delta u=0$ is denoted by $g(h), e t c$.
§3. $O_{\Omega}, O_{\omega}, O_{B}$ and $O_{G}$. that

In this section we shall prove

$$
O_{G} \subset O_{\omega}=O_{\Omega}=O_{B} .
$$

Theorem 3.1. If $F \in O_{\Omega}$, then $F \in O_{B}$, and vice versa.

Proof. Il $u(z)$ is a bounded solution of (A) vanishing not identically, then, by Lemma 2.1 ,

$$
m \Omega_{n}\left(z, \Gamma_{n}, F_{n}\right) \leqq u(z) \leqq M \Omega_{n}\left(z, \Gamma_{n}, F_{n}\right),
$$

where $M=\sup _{F} u(z)$

$$
, m=\inf _{F} u(z)
$$

Letting $n$ tend to $\infty$, we have

$m \Omega(x, \Gamma, F) \leqq u(z) \leqq M \Omega(z, \Gamma, F)$.

If $F \in O \Omega$, then $\Omega(z, \Gamma, F)$

and therefore $u(z)$ is identically zero. This contradicts $u(z) \neq 0$.

Conversely, if we assume $F \notin O_{\Omega}$, then $\Omega(z, \Gamma, F) \quad$ is a bounded solution of $(A)$ and $\neq 0$.

$$
F \frac{\text { Theorem 3,2. }}{\epsilon O_{\omega} \cdot} \text { If } F \in O_{G} \text {, then }
$$

Proof. In order to prove the theorem, we need some lemmata:

Lemma 3.1. $F \in O_{\omega}^{(h)} \rightarrow F \in O_{\omega}$.

Lemma 3.2. $F \in O_{G} \rightarrow F \in O_{G}^{(h)}$.

Lemma 3.3. $F \in O_{\omega}^{(h)} \rightleftarrows F \in O_{G}^{(h)}$.

Proof of Lermata 3.1 and 3.2 . By ihe Lemma 2.2, we have

$$
\omega_{n}\left(x, \Gamma_{n}, F_{n}-F_{0}\right) \leqq \omega_{n}^{(k)}\left(z, \Gamma_{n}, F_{n}-F_{0}\right)
$$

and

$$
g_{n}\left(z, x_{0}\right) \leqq g_{n}^{(n)}\left(x, z_{0}\right),
$$

from which we obtain the desired result.

Lemma 3.3 is a well-known result. $q \cdot \theta \cdot d$

Thus we have $O_{G} \subset O_{G}^{(h)}=O_{\omega}^{(h)} \subset O_{\omega}$.

By Lemma 3.2 , we deduce the followIng property: If the boundary of contains a continuum, then $F \notin O_{G}$.

Remark. The Definition 2.12 (a) is very artificial in the sense that 


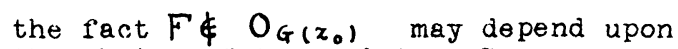
the cholce of the point

In the harmonic case, $F \notin O_{G\left(x_{0}\right)}^{(h)}$ is equivalent to $F \notin O_{G}^{(h)}$, but in cur case we can not yet conclude whether $F$ F $O_{G\left(x_{0}\right)}$ is equivalent to

F $O_{G}$ or not. Here we shall explain a surficient condition in

order that $F \notin O_{G\left(z_{0}\right)}$ implies

$F \notin O_{G}$

If $\Omega(z) \geq \varepsilon>0$ f'or all points $z$ on $F$, then $F \notin O_{G\left(z_{*}\right) \text { im- }}$ plies $F \notin O_{G}$.

Proof. Let $\Gamma_{0}$ surround a compact connected analytic subregion $F_{0}$, such that $z_{0}, z_{0}^{\prime} \in F_{0}$. Then we can choose two points $p_{m}, p_{m}^{\prime}$ on $\Gamma_{0}$, such that

$$
g_{m}\left(p_{m}, z_{0}\right)=\frac{2 \pi \Omega_{m}\left(z_{0}\right)}{-\int_{\Gamma_{0}} \frac{\partial}{\partial \nu} \omega_{m}(x) d s}
$$

and

$$
g_{m}\left(p_{m}^{\prime}, z_{0}^{\prime}\right)=\frac{2 \pi \Omega_{m}\left(x_{0}^{\prime}\right)}{-\int_{\Gamma_{0}} \frac{\partial}{\partial v} \omega_{m}(x) d s},
$$

because, by Green's formula, we have

$$
-\int_{\Gamma_{0}} g_{m}\left(x, z_{0}\right) \frac{\partial}{\partial y} \omega_{m}(x) d s=2 \pi \Omega_{m}\left(z_{0}\right) \text {. }
$$

Thus we have

$$
\frac{g_{m}\left(p_{m}, z_{0}\right)}{\Omega_{m}\left(z_{0}\right)}=\frac{g_{m}\left(p_{m}^{\prime}, z_{0}^{\prime}\right)}{\Omega_{m}\left(z_{0}^{\prime}\right)},
$$

from which we get the desired result.

Theorem 3.3. If $F \in O_{\Omega}$, then $F \in O_{w}$ and vice versa.

Proof. Necessity. By Lemma 2.1, we have

$$
\Omega_{n}\left(z, \Gamma_{n}, F_{n}\right) \geqq \omega_{n}\left(x, \Gamma_{n}, F_{n}-F_{0}\right), \quad z \in F_{n}-F_{0},
$$

thus, by $n \rightarrow \infty$, we conclude the
necessity.

In order to prove the sufflciency, we need some preperatory lemmata.

Lerma 3.4. If $F \notin O_{\Omega}$ and

$\in O_{\omega}$, then $F \in O_{G}$.

Lerma 3.5 . If $F \notin O_{\Omega}$, then

$\sup _{F} \Omega(z, F)=1$

Lemma 3.6. If $F \in O_{\omega}^{(k)}$, then

$F \in O_{\Omega}$.
Proof ol Lemma 3.4. By Green's Iormula, we have

$$
g_{m}\left(p_{m}, z_{0}\right)=\frac{2 \pi \Omega_{m}\left(z_{0}\right)}{-\int_{\Gamma_{0}} \frac{\partial}{\partial \nu} \omega_{m}(z) d s} .
$$

If $F \in O \omega$ and $\& O_{\Omega}$, then $\omega_{m} \rightarrow 0$, and hence $\partial \omega_{m} / \partial \nu \rightarrow 0$ on $\Gamma_{0}$. We may assume that $\Omega_{m}\left(z_{0}\right)$ $\neq 0$ and $\Omega\left(z_{0}\right) \neq 0$, and thus $g_{m}\left(p_{m}, z_{0}\right) \rightarrow \infty$. Theretore $g\left(x, z_{0}\right)=\infty$ for a point $z \neq z_{0}$.

Proof of Lemma 3.5. Let $\sup _{\mathrm{F}} \Omega(z, F)=\lambda$, then $\lambda \leqq 1$ is evident, and thus we shall prove $\lambda \geqq 1$. For $z \in F_{n}$, we shall consider the function

$g_{n}(z)=\Omega_{n}\left(z, F_{n}\right) S_{n p} \Omega(z, F)-\Omega(z, F)$ then $\Delta \varphi_{n}(z)=P \Phi_{n}(z) \quad$ for $z \in F_{n}$, and $\varphi_{n}(x) \geq 0$ on $\Gamma_{n}$. Therefore, from Lernma 2.1 , we have $\Phi_{n}(x) \geqq 0$ on $F_{n}$, and hence

$$
\Omega_{n}\left(z, F_{n}\right) \sup _{F} \Omega(z, F) \geq \Omega(z, F)
$$

$$
\text { on } \begin{aligned}
& F_{n} \quad \text { Letting } n \rightarrow \infty, \\
& \lambda \Omega(z, F) \geqq \Omega(z, F)(\geqq 0, \neq 0),
\end{aligned}
$$

from which $\lambda \geqq 1$. q.e.d.

Since $\Omega(z, F) \quad$ is a bounded non-negative solution of $(A)$ on $F$, we have

$$
\sup _{F} \Omega(z, F)=\sup _{F-R} \Omega(x, F) \text {, }
$$

where $R$ is an arbitrary compact analytic subregion of $F$.

Proof of Lemma 3.6. Suppose that $F$ does not belong to $O_{\Omega}$, but belongs to $O_{\omega}^{(h)}$, then we have, by Lemma $3.5, \sup \Omega(x, F)=\sup _{F} \Omega(x, F)$ $=1$, and $\sup _{F \rightarrow F_{0}} \omega^{(h)}\left(x, F-F_{0} F_{0}\right)=0$.

Let $g_{n}(x)=\Omega_{n}\left(x, F_{n}\right)-\omega_{n}^{(k)}\left(z, F_{n}-F_{0}\right)$ for $z \in F_{n}-F_{0}$, then $\Delta \varphi_{n}(z)$ $=P \Omega_{n}\left(z, F_{n}\right) \geq 0$, and hence $\sup _{\Gamma_{n}+\Gamma_{0}} g_{n}(z)$ $=\sup _{F_{n}-F_{0}} g_{n}(z)$. On the other hand, $\varphi_{n}(x)=0$ on $\Gamma_{n}$ and $\Gamma_{0}$. Thus $\Gamma_{0}$, but $\$ 0$ on $\sup _{F_{n}-F_{0}} \varphi_{n}(x)=\sup _{\Gamma_{0}} \varphi_{n}(z)=\sup _{\Gamma_{0}} \Omega_{n}\left(z, F_{n}\right)$, ${ }^{\text {or }} \Omega_{n}\left(z, F_{n}\right) \leqq \sup _{\Gamma_{0}} \Omega_{n}\left(z, F_{n}\right)+w_{n}^{(h)}\left(z, F_{n}-F_{0}\right)$ 
Letting $n \rightarrow \infty$, wa hame

$\Omega(x, F) \leq \sup _{\Gamma_{0}} \Omega(z, f)+\omega^{(R)}\left(z, F-F_{0}\right)$,

and therefore

$$
\begin{aligned}
\sup _{F} \Omega(z, F) & =\sup _{F-F_{0}} \Omega(z, F) \\
& \leq \sup _{\Gamma_{0}} \Omega(z, F)+\sup _{F-F_{0}} \omega^{(h)}\left(x, F-F_{0}\right) .
\end{aligned}
$$

Considering our first assurptions, we have

$$
1 \leqq \sup _{\Gamma_{0}} \Omega(x, F) \text {, }
$$

Which contradicts Lemma 2.3 and the monotone decreasing property of $\Omega_{n}(z, F)$, that is, $0 \leqq \Omega_{n}\left(z, F_{n}\right)$ $1\left(x, F_{m}\right) \leqq \Omega_{n}\left(z, F_{n}\right)^{m} \geq n$ and hénce $\begin{array}{ll}\Omega_{m}\left(x, F_{m}\right) \leqq \Omega_{n}\left(z, F_{n}\right) & \text { and hence } \\ \sup _{(2, F) \leqslant 1} \text {, wich is absurd }\end{array}$ $\sup _{0} \Omega(z, F) 1$, which is absurd. $q \cdot e \cdot d$.

Sulficlency proof of Theorem 3.3 . Suppose that $F \in O \omega$ and $F \notin O \Omega$, then by Lemma 3.4 $F \in O \&$ and hence, by Lemmata 3.2 and $3.3, F \in O O_{\omega}^{(k)}$.

Thus $F \in O \Omega$ by Lemma 3.6, which is a contradiction, q.e.d.

8. Subregions and $O_{B}$.

Let $G$ be a non-compact connected subregion, and its relative boundary $C$ consist of a finite number of analytic curves. Supposing that $\left\{F_{n}\right\}, n=0,1, \ldots$ is an exhaus tion of $F$, we introduce the notations: $G_{n}=F_{n} G, \gamma_{n}=\Gamma_{n} n G$ and $C_{n}=F_{n} \cap C^{n}$.

Definition 4.1. Let $\omega_{n}\left(x, G_{n}\right)$, $\Omega_{n}\left(z, \hat{G}_{n}\right)$ and $\omega_{n}^{\prime}\left(x, G_{n}\right)$ be the solutions of (A) on $G_{n}$, sttisfying the following boundary oonditions :

$$
\begin{aligned}
& \omega_{n}\left(x, G_{n}\right)= \begin{cases}0 & \text { on } C_{n} \\
1 & \text { on } \gamma_{n} ;\end{cases} \\
& \Omega_{n}\left(z, G_{n}\right)=1 \text { on } C_{n}+\gamma_{n} \text {; }
\end{aligned}
$$

and

$$
\omega_{n}^{\prime}\left(x, G_{n}\right)=\left\{\begin{array}{lll}
0 & \text { on } \gamma_{n}, \\
1 & \text { on } C_{n} .
\end{array}\right.
$$

rospectively.

Evident].y we nave $\Omega_{n}\left(z, G_{n}\right)=\omega_{n}\left(x, G_{x}\right)$ $+\omega_{n}^{\prime}\left(x, G_{n}\right)$, and, from Lemma 2.1 , we have the rolizowing monotonalty: ror $m \geq n$

$$
\omega_{n}\left(z, G_{m}\right) \leqq \omega_{n}\left(x, G_{n}\right) \text {, }
$$

$$
\Omega_{m}\left(x, G_{m}\right) \leqq \Omega_{n}\left(x, G_{n}\right)
$$

and

$$
\omega_{m}^{\prime}\left(z, G_{m}\right) \geqq \omega_{n}^{\prime}\left(z, G_{n}\right)
$$

Hence, from the unf form boundedness of these quantities; the ilmits

$$
\begin{aligned}
& \lim _{n \rightarrow \infty} \omega_{n}\left(x, G_{n}\right)=\omega(x, G), \\
& \lim _{n \rightarrow \infty} \Omega_{n}\left(x, G_{n}\right)=\Delta(x, G) .
\end{aligned}
$$

and

$$
\lim _{n \rightarrow \infty} \omega_{n}^{\prime}\left(z, G_{n}\right)=\omega^{\prime}(z, G)
$$

exist, and moreover we have $\Omega(x, G)$ $=w(x, G)+w^{\prime}(x, G)$.

Definition 4.2. $G \in \mathrm{SO}_{\omega}$ means that $\omega(z, G)=0$.

Evidently $G \in S O \omega$ is aquivalent to $\boldsymbol{\Omega ( x , G )}=\boldsymbol{\omega}^{\prime}(z, G)$.

If (z) is a bounded nonconstant solution of $(A)$, being continuous on $G+C$ and $u(x)=h$ \&const.j on $C$, then get

$$
\begin{aligned}
& h \Omega(x, G)-M w(x, G) \leqq u(z) \\
& \leq k \Omega(x, G)+M \omega(x, G),
\end{aligned}
$$

where $M=\sup |u(z)-k \Omega(z, G)|$ This is easily verifled by means of Lomma 2.1 tor $G_{n}$. Let $n$ tend to $\infty$.

If $\omega(x, G) \equiv 0$, then $\mu(x)$ $=h \Omega(x, G)$. Even if $\omega(x, G) \equiv 0$ there is at least a bounded nonconstant solution $u(x)$ of $(A)$, satisfying the condition $u(z)=$ const. $(\neq 0)$ on $C$, but, indeed, essentially only one, that is, $\Omega(z, G)$. If $\omega(z, G) \neq$ then there is at least two bounded, non-constant solutions $u(x)$ of (A), satisfying the condition $u(z)=$ const. $(\neq 0)$ on $C$, and being Inearly independent, for example, $k \Omega(z, G)$ and $h \Omega(z, G)$ $+M \omega(x, G)$.

Theorem 4.1. $G \in S O \omega$ is oquivalent that there is only one Iinearly independent solution $\Omega(z, G)$ of (A) bounded ron-constant in $G$ and satistying the condition $\Omega(z, G)$ $=1$ on $C$. $G \in S O \omega$ is equi- 
valent that there is no bounded nonconstant solution of (A) vanishing identically on $C$.

Theorem 4.E. In order to $F \in O_{B}$ it is necessary and sut'licient that there is at least a non-compact connected subregion $G$ which does not belong to $\mathrm{SO}_{\mathrm{w}}$.

Proof. Sufficiency. Let $u_{n}(x)$ be a finite solution of (A) on $F_{n}$ such that

$$
u_{n}= \begin{cases}1 & \text { on } \gamma_{n}, \\ 0 & \text { on } \Gamma_{n}-\gamma_{n},\end{cases}
$$

then $0 \leqq u_{n}(z) \leqq 1$ on $F_{n}$, by Lemma 2.1 .

$$
u_{n}(z) \text { being uniformly bounded, }
$$
we can select a subsequence $\left\{u_{n_{v}}(z)\right\}$ of $\left\{u_{n}(x)\right\}$ which converges to a bounded solution $u(z)$ of (A) on $G$. For the sake of simplicity we shall retain the original suffices.

By Lemma 2.1, we have $u_{n}(z) \geq \omega_{n}\left(z, G_{n}\right)$ on $G_{n}$ - Let $n$ tend to $\infty$ ave $u(z) z \omega(z, G)$. By the as sumption $G \notin S O_{\omega}, \omega(z, G) \neq 0$. Hence there exists a point $z_{0}$ on $G$, at which $\omega\left(z_{0}, G\right)>0$. Thus $u\left(z_{0}\right)>0$. Hence $u(z) \neq 0$ and is not constant.

Necessity. By the definition of $F \notin O_{B}$, there is a bounded non-constent solution $u(z)$ of (A) on $F$. Then there exists a point $z_{0}$ on $F$ at which $u\left(z_{0}\right) \neq 0$.

(a) If $u\left(z_{0}\right)>0$, then we take $G$ as the point-set on which $u(z)>u\left(z_{0}\right)$.

(b) If $u\left(z_{0}\right)<0$, then we take $G$ as the point-set on which $u(x)<u\left(z_{0}\right)$.

We shall treat here only the case (a), since the case (b) is similar.

If $G$ has a compact connected component $G_{\nu}, u(x)$ is subharmonic in $G_{\nu}$, thus by Lemma $2.1 \quad \sup _{C_{\nu}} u(z)=\sup _{\nu} u(z)>0$, where

$C_{v}$ is the boundary of $G_{\nu}$. on the other hand $\sup _{u}(z)=u\left(z_{0}\right)$,

which is absurd. Thus $G$ must have a non-compact connected subregion. Obviously we have $u(z) \neq u\left(z_{0}\right)$. $\Omega(z, G)$ on $G$, and $u(z)$ is a bounded solution of (A) on $G$, with constant boundary value $u\left(z_{0}\right)$ on
C. By Theorem 4.I, G\&SOw. $q \cdot e \cdot d$.

Theorem 4.3. (1) If $G$ is a connected subregion of $F$, having Jordan relative boundary $C$, and $u(z)$ is a solution of $(A)$ continuous on $G+C$. Suppose that $F \in O_{3}$ and $u(z)$ is bounded, non-negative and non-constant on $G$, then the maximum principle holds, that is,

$$
\sup _{G} u(x)=\sup _{C} u(z) \text {. }
$$

(ii) If $u(z)$ is non-positive and the above assumptions remain valid, then the minimum principle holds, that is,

$$
\inf _{G} u(z)=\inf _{C} u(z) \text {. }
$$

Proof. We shall prove the flrst part of the theorerl, as remaining part can be proved similarly.

If' $G$ is a compact subregion, the theorem is evident. Thus we shall contine ourselves to a non-compact subregion. If we suppose that sup $u(z)$ $+\sup _{G} u(z)$, then there is a positive number $M$, such that

$$
0 \leqq \sup _{C} u(x)<M<\sup _{G} u(z) \text {. }
$$

Let $H$ be a point-set on which $u(z)>M$ holds, and $H_{\nu}$ be a connected component of $H$. $H_{\nu}$ is a connected subregion with a noncompact closure. This liact is shown by Lemma 2.I. Now $u(x)>M$ in $H_{y}$ and $M \Omega\left(z, H_{v}\right)$, since $M \Omega\left(z, H_{v}\right) \leqq M$ in $H_{v}$; Hence, by Theorem 4.I, $\omega(x, H) \neq 0$. By Theorem 4.2, $F \notin O_{B}$, which is absurd.

Theorem 4.31. If $F-G$ is compact, then the converse of the Theorem 4.3 holds.

Proof. Let $u(z)$ be a boundea non-negative solution of (A) on F. II we suppose $\sup _{G} u(z)=\sup _{\text {ct }} u(z)$, tinen Sup $u(x)$ is attained on $C$. Thus $u(z)$, as a function defined on $\bar{F}$, has its maximum on F.- G This is contradictory. Thus $u(z) \equiv 0$, that is, $F \in O_{B}$.

Theorem 4.4. Let $G$ be a noncompact connected subregion, having 
a Jordan relative boundary $C$. Then $G \notin S O \omega$ is a necessary and sufficient condition in order that the maximum principle does not hold on $G$.

Proof. Surficiency was already proved in the Theorem 4.3 .

Necessity. Suppose that $G \notin S O_{\omega}$ and the maximum princlple holds. Then $\boldsymbol{\omega}(z, G)$ is a bounded non-constant solution of (A) on $G$. Thus

$$
\sup _{G} \omega(x, G)=\sup _{C} \omega(x, G)=0,
$$

and hence $\omega(x, G)=0$ on $G$, which is absurd.

55. Subregions, $O_{D}$ and $O_{K}$.

Theorem 5.1. $O_{\tau} \rightleftarrows O_{K}$.

Proof. We first show that $O_{K}$ $\rightarrow O_{D}$ suppose that $F \& O_{D}$, then there is a function $\varphi(x)$ $\epsilon L^{2}(F)$ and $g \neq 0$. Let

$$
u(z)= \begin{cases}\varphi(x), & z \in F_{n}, \\ 0, & z \in F-F_{n},\end{cases}
$$

then we may assume that $u\left(z_{0}\right) \neq 0$, where $z_{0}$ is a fixed point on $F_{n}$ : By the reproducing property of the kernel and the Schwarz's Inequality, we have

$$
\begin{aligned}
0 \leqslant\left|u\left(z_{0}\right)\right|^{2} & \leqq D_{F_{n}}\left(K_{n}\left(z, z_{0}\right)\right) D_{F_{n}}(u(z)) \\
& =K_{n}\left(z_{0}, z_{0}\right) D_{F_{n}}(u(x)) .
\end{aligned}
$$

Let $n$ tend to $\infty$, then we have

$$
0 \lessgtr\left|\varphi\left(z_{0}\right)\right|^{2} \leqslant K\left(z_{0}, z_{0}\right) D_{F}(\varphi(z)) .
$$

$$
\begin{aligned}
\text { Thus } & K\left(x_{0}, x_{0}\right) \neq 0 \text {, which shows } \\
F & \neq O_{K}
\end{aligned}
$$

We next show that $O_{D} \rightarrow O_{K}$. Let $F \notin O_{K}$, then we can choose a point $z_{0} \in F$ such that $K\left(z_{0}, z_{0}\right)$ $\neq 0,+\infty$, that is, we have $\infty$ $>M^{\prime}>K_{n}\left(z_{0}, z_{0}\right)>\varepsilon>0$ for a surficiently large integer $n, M$ and $\varepsilon$ being independent of $n$. Now the Iimit $\lim _{x \rightarrow \infty} K_{n}\left(z_{0}, x_{0}\right)=K\left(z_{0}, z_{0}\right)$ exists. Evidently $D_{F_{n}}\left(K_{n}\left(z_{0}, z_{0}\right)\right)$ $=K_{n}\left(z_{0}, z_{0}\right)<M$. Thus $K\left(x, z_{0}\right) \in L^{2}(F)$.

Corollary. Let $E$ be a compact set on $F_{1}$, being 1 tself a compact subregion of $F$, and $\varphi(z)$ be an arbitrary Dirichlet-finite solution of (A) in $F_{1}-E$. Then a necessary and sulficient condition in order that $\varphi(x)$ is prolongable onto $E$ in the sense of (A) is that

$$
K_{F_{1}-E}(z, z)=K_{F_{l}}(z, z) \text {. }
$$

For later usages we shall explain an extremal property of the sclution of (A).

Let $R$ be a compact connected subregion of $F$, surrounded by compact analytic curves $C$. If $u(z)$ satisties the following properties, then we write $u(z) \in \mathcal{G}_{R}$

1) $u(z)$ is a continuous differentiable function in $R$,

2) $u(z)$ has a given boundary function $\varphi(z)$ being continuousiy differentiable on $C$ except only at a finite number of points.

Lemma 5.1. If $v(z)$ is a sclution of (A) with the boundary value

$\varphi(z)$, then $D_{R}(v) \leqq D_{R}(u)$ for any $u \in \mathcal{G}_{R}$.

Proof. We put $u(z)=v(z)+\Phi(z)$ then $\Phi(z)$ vanishes everywher' on $C$, and is continuousiy difterentiable on $G+C$, except only at a finite number of boundary points. Thus have from the Green's formula

$$
D_{R}(v, \Phi)=\left\{\Phi \frac{\partial v}{\partial v} d s=0 .\right.
$$

Hence we have $D_{R}(u)=D_{R}(v)+D_{R}(\Phi) \geq D_{R}(v)$. $q \cdot e \cdot d \cdot$

Let $G$ be a non-compact connected subregion of $F$ with an analytic, but not necessarily compact, relative boundary $C$. Notations $G_{m}$,

$C_{m}, \gamma_{m}$ and others are the sare a's those defined in the preceding section.

Lemma 5.2. There exists a fundamental solution $\hat{N}_{m}(z, x)$ of (A), such that $\hat{N}_{m}(z, x)=0$ on $C_{m}$ and $\frac{z}{\partial v} \hat{N}_{m}(z, x)=0$ on

Proof He may asure that Gm is any canonical domain, for example, $\gamma \mathrm{m}$ consf.sts of a linite number or segments on the real axis, because $P(z)$ has the covariance character and hence Dirichlet integral, Green function, ete. with regard to the differential equation (A) remain invarlant by conformal mapping.

Let $\widetilde{G}_{m}$ and $\widetilde{z}$ be the inversions of $G_{m}$ and $z$ with respect to $\gamma_{m}$, respectively. And then we identify $G_{m}$ and $\widetilde{G}_{m}$ by all corresponding segments $\gamma_{m}$. such a domain is denoted by $U^{\prime}$ that is, Horo. 
over we define that $P(\widetilde{z})=P(z)$

for $\widetilde{z} \in \widetilde{G}_{m}$ when we construct the following solution of $(A)$ :

$$
\hat{N}_{m}(z, x)=g_{\frac{c}{G}}(z, x)+g_{G_{m}}(z, \tilde{x}), \quad x \in G_{m} .
$$

Then $\hat{N}_{m}(x, x)$ is a desired solution of $(A)$. In l'act, we have evidently

$$
g_{\overline{G_{m}}}(z, x)=g_{G_{m}}(\tilde{z}, \tilde{x}),
$$

and hence

$$
\hat{N}_{m}(z, x)=\hat{N}_{m}(\tilde{z}, \tilde{x})=\hat{N}_{m}(\tilde{z}, x) \text {. }
$$

Thus

$$
\frac{\partial}{\partial y} \hat{N}_{m}(z, x)=0, \quad I_{m} z=y \text { on } \gamma_{m} \text {. }
$$

on the other hand, we have $\hat{N}_{m}(z, x)$ $=0$ on $C_{m}$ and $\hat{N}_{m}(x, x)$ is a fundamental solution of $(A)$ on $G_{m}$.

Unlqueness can be proved as follows :

Let $\hat{L}$ and $\hat{N}$ be two desired Iunctions, then $\hat{L}-\hat{N}=9$ is a rinite solution of (A) satisting the condition: $\rho=0$ on $C_{-}$ and $\frac{3}{\partial r} \varphi=0$ on $\gamma_{m}$. By Green's formula we have

$$
\begin{aligned}
D_{G_{m}}(\varphi) & =-\int_{C_{m}+\gamma_{m}} \varphi \frac{\partial}{\partial v} \varphi d s-\iint_{G_{m}} \varphi[\Delta \rho-p \rho] d x d y \\
& =0 .
\end{aligned}
$$

Thus $\hat{L}^{\Phi} \equiv \hat{\mathrm{N}}^{0}$ on $G_{m}$. , that

Let $g_{m}(z, x)$ be the Green I'unction of $(A)$ on $G_{m}$, then $\lim _{m \rightarrow \infty} g_{m}(z, x) \neq \infty \quad$ for $z(+x) \in G$ was proved in $\$ 3$. Th1s will be made use or in later Lema 5.4 .

By the Groen's formula we have $D_{g_{m}}\left(u, \gamma_{m}\right)=0$ and $D_{G_{m}}(u(x)$ $\left.\hat{N}_{m}(x, x)\right)=2 \pi u(x)$, where $u(x)$ is an arbitrary Dirighlet finite solution of (A) on $G_{m}$ ith continuous boundary values, such that

$u(x)=0$ on $C_{m}$. This family is denoted by $L_{0}^{2}\left(G_{m}\right)$. That the runction

$$
\hat{K}_{m}(z, x)=\frac{1}{2 \pi}\left(\hat{N}_{m}(x, x)-g_{m}(x, x)\right)
$$

is the reproducing kernel function of $L_{0}^{2}\left(G_{m}\right)$ is an immodiate consequence of the above two relations. $\underset{\text { for } a \operatorname{Lix\theta d}}{\operatorname{Lemma} 5.3 .} \hat{K}_{m}(z, x) \geq 0$ on $G_{m}$

Proof. From the manner of construction of $\hat{N}_{m}(z, x)$, this proposition is evident, but we shall explain another proof, being similar to that in a Bergman-Schiffer's paper [2] $\dot{G}$ Suppose that, for a rixed $x \in G_{m}$, $\hat{N}_{-}(z, x)$. Let negative somewhere in $G_{m}{ }^{\circ} \hat{N}_{m}<0$ and $b^{-}$be its boundary; evidently $G_{m}^{-}$is a subregion of $G_{m}$, $b^{-}$ consists either of smonth arcss of $C_{m}+\gamma_{m}$ on whi ch $\hat{N}_{m} \frac{\partial}{\partial v} \hat{N}_{-}=0$ or of smooth level curves $\hat{N}_{m}(z, x)$ $=0$. Then $D_{G=}\left(\hat{N}_{m}(z, x)\right)$ exists, because the pole $x$ of $\hat{N}_{m}(x, x)$ does not lie in $G_{m}^{-}$, and evidently $>0$. By the Green's tormula, we have

$$
\begin{aligned}
0 & \neq D_{G_{m}^{-}}\left(\hat{N}_{m}(x, x)\right) \\
& =-\int_{b^{-}} \hat{N}_{m}(x, x) \frac{\partial}{\partial \nu} \hat{N}_{m}(x, x) d s \\
& =0,
\end{aligned}
$$

which is absurd. Thus $\hat{N}_{m}(z, x) \geq 0$ on $\gamma_{m}+C_{m}+\dot{G}_{m}$. On the other hand, $g_{m}(z, x)=0$ on $\gamma_{m}+C$, and therefore $\hat{N}_{m}(z, x)-g_{m}(x, x) \geq 0$ on $\gamma_{m}+C_{m}$, and hence, by Lemma 2.1, we have

$$
\begin{aligned}
\hat{K}_{m}(x, x) & =\frac{1}{2 x}\left(\hat{N}_{m}(x, x)-g_{m}\left(x, x_{i}\right)\right. \\
& \geqq 0
\end{aligned}
$$

on $G_{m}+C_{m}+\gamma_{m}$.

Lemma 5.4. Let $G$ be a non-compact connected subregion of $F$ with an analytic relative boundary $C$. If there exists a non-constant solution $u(z)$ of $(A)$ on the closure $\bar{G}$ of $G$, such that $D_{G}(u)<\infty$ and $u(z)=0$ on $C$, then there exists a non-constant bounded nonnegative and Dirichlet-finite solu$\begin{array}{lll}\text { tion } v(z) & \text { of }(A) \text { on } G+C \\ \text { such that } & v(z)=0 & \text { on } C\end{array}$

Proof. This is an analogue of Morl's Lemma [1], stating the same I'act as in the harmonic case; Let $u(z)$ belong to $L_{0}^{2}(G)$, and be non-constant. Evidently $u(z) \in L_{0}^{2}\left(G_{m}\right)$. Thus $D_{\alpha_{m}}\left(u(x), \hat{R}_{m}^{0}(z, x)\right)=u(x)$. Moreover $\hat{K}_{n=v e}(z, x) \in L_{0}^{2}\left(G_{m}\right)$, theretore wo

$$
D_{G_{m}}\left(\hat{K}_{m}(z, z), \hat{K}_{m}(z, x)\right)=\hat{K}_{m}(x, x) \text {. }
$$


For $m \geqq n$, we obtain

$$
D_{G_{n}}\left(\hat{K}_{m}(z, x), \hat{K}_{n}(z, x)\right)=\hat{K}_{m}(x, x) \text {, }
$$

anci hence

$$
\begin{aligned}
0 & \leqq D_{G_{n}}\left(\hat{K}_{m}-\hat{K}_{n}\right) \\
& =D_{G_{n}}\left(\hat{K}_{m}\right)-2 D_{G_{n}}\left(\hat{K}_{m}, \hat{K}_{n}\right)+D_{G_{n}}\left(\hat{K}_{n}\right) \\
& \leqq D_{G_{m}}\left(\hat{K}_{m}\right)-2 D_{G_{n}}\left(\hat{K}_{m}, \hat{K}_{n}\right)+\hat{K}_{n}(x, x) \\
& =\hat{K}_{m}(x, x)-2 \hat{K}_{m}(x, x)+\hat{K}_{n}(x, x) \\
& =\hat{K}_{n}(x, x)-\hat{K}_{m}(x, x) .
\end{aligned}
$$

Thus, Ior $m \geqq n$,

$$
0 \leqq \hat{K}_{m}(x, x) \leqq \hat{K}_{n}(x, x) \text {, }
$$

and hence

$$
\left.\lim _{n \rightarrow \infty} \hat{K}_{n} i x, x\right)=\hat{k}(x, x),(\geqq 0)
$$

exists. Tinus

$$
\lim _{m>r_{1} \rightarrow \infty} D_{G_{n}}\left(\hat{K}_{m}-K_{*}\right)=0
$$

and simultaneously

$$
\lim _{m \rightarrow n \rightarrow \infty} D_{G_{n}}\left(u(x), \hat{K}_{m}(x, x)-\hat{K}_{n}(x, x)\right)=0 \text {. }
$$

Thus

$$
\lim _{n \rightarrow \infty} D_{G_{n}}\left(u(x), \hat{K}_{n}(z, x)\right)
$$

exists and is equal. to $u(x)$. If we choose a point $x$ such that $u(x) \neq 0$, then we have

$$
|u(x)|^{2} \leqq \hat{K}_{n}(x, x) D_{G_{n}}(u) \leqq \hat{K}_{n}(x, x) D(u) .
$$

Thus $\infty>\hat{K}(x, x)>0$

this and the Schwarz's inequality we see that $\lim _{n \rightarrow \infty} \hat{K}_{n}(z, x)$ exists on a compact subregion of $G$. Moreover, from

$$
D_{G_{n}}\left(\hat{K}_{n}(x, x)\right)=\hat{K}_{n}(x, x),
$$

we see that $D_{G}\left(\hat{K}_{2}(z, x)\right)<\infty$, and

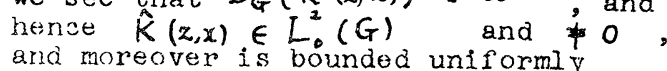
in $G$ in a wider sense.

Renaining part of the Lemma is that $K(z, x)$ is a bounded solution of (A) on $G$. To prove this we remark that $g_{m}(z, x) \neq \infty$ for all points $z(\neq x) \in G$ Let $\Gamma_{0}$ be an analytic compact curve in $G$, surrounding a compact domain $\left.{ }_{\hat{N}} G_{0}, \dot{x}\right)$ in which the point $x$ lies. $\hat{N}_{m}(z, x)$ is of non-negative vilue on $\gamma_{m}$ other hand, on $\gamma_{m}$

$$
\frac{\partial}{\partial \nu} \hat{N}_{m}(z, x)=0 \text {, }
$$

thus

$$
\sup _{G_{m}-G_{0}} \hat{N}_{m}(x, x)>\max _{\gamma_{m}} \hat{N}_{m}(x, x)
$$

$\begin{array}{lll}\text { vecause, if } & \hat{N}_{m}(x, x) & \text { has its } \\ \text { Sup }_{m} G_{0} & \text { on } \gamma_{m} \text {, then } \hat{N}_{m}(x, x)\end{array}$ as a function on $\overline{\bar{G}}_{m}-\bar{G}_{0}$, has a maximum at an inner point on $G_{m}$ harmonicity of $\hat{N}_{m}(z, x)$, where $\bar{G}_{0} \quad$ is the union of two domains $G_{0}$ and $\widetilde{G}_{0}$, $\widetilde{G}_{0}$ being the inversion domain of $G_{0}$ with respect to $\gamma_{m}$. Since $\hat{N}_{m}(z, x) \equiv 0$ on $\widehat{N}_{m}^{m}(x, x)$ from the subharmonicity of $\sup _{G_{m}-G_{0}} \hat{N}_{m}(z, x)=\max _{\Gamma_{0}} \hat{N}_{m}(x, x)$. Thus, for $z \in G_{m}-G_{0}$,

$$
\begin{aligned}
\lim _{m \rightarrow \infty} \hat{N}_{m}(x, x) \leqq \lim _{m \rightarrow \infty} \sup _{\Gamma_{0}} \hat{N}_{m}(x, x) \\
=\lim _{m \rightarrow \infty} \sup _{\Gamma_{0}}\left(2 \pi \hat{K}_{m}(z, x)-g_{m}(x, x)\right)
\end{aligned}
$$$$
<\infty \text {. }
$$

Thus $\hat{N}(x, x)$ is boundea on $G-G_{0}$ - Un the other hand, $\hat{N}(z, x)-g(x, x)$
$G_{0}$ is bounded on bounded on $G_{0}$ and $G-G_{0}$, and hence on $G$. q.e.d.

Theorem 5.1. Let $G$ be a noncompact connected subregion of $F$ with an analytic relative boundary C . If there exists a non-constant solution $U(z)$ of (A) on $G+C$, such that $U=0$ on $C$ and $D_{G}(U)<\infty$ conversely, if Fi can find such a domain $O_{D}$, then a solution $U(x)$ of $(A)$.

Proof. By the Lemma 5.4, we may assume that $0 \leqq U(z) \leqq 1$ in $G$, and $\sup _{G(z)=1}$, Let $V(z)$ be solution of $(\dot{A})$, such that $V(x)=U(z)$ on $G,=0$ on $F-G$. $V(z)$ is continuous on $F$ and has plecewise continuous partial derivatives, and $D_{F}(V)=D_{G}(U)<+\infty$. 


\begin{abstract}
Let $u_{x}(z)$ be a solution
$F_{n}$, sucn that $u_{n}(z)$
$=V(x)$ on $\Gamma_{n}$ : Since $u_{x}(x)^{x}$

is uniformly bounded solution of $(A)$. we may assume that $u_{n}(z)$ converges to a solution $\mu(z)$ uniformly in the wider anse on $F$.
\end{abstract}

For $m \geq n$, we have, by the Lemma 5.1,

$$
D_{F_{n}}\left(u_{m}\right) \leqq D_{F_{m}}\left(u_{m}\right) \leqq D_{F_{m}}(V) \leqq D_{F}(V) .
$$

Letting eirst on tend to $\infty$ and next $n$ to $\infty$, we have

$$
D_{F}(u) \leqq D_{F}(V)<+\infty \text {. }
$$

Let $H$ be a subregion of $G$, on which $U(x ;>1 / 2$. $H$ has 8 non-compact connected component $H_{\nu}$. In $H_{v}, 1 \geqq U(x)>1 / 2$, thus $U(z)+\frac{1}{2} \Omega_{\left(z, H_{\nu}\right)} 0^{\circ}$ Hence, by Theorem $4.1, H_{\nu} \notin 50_{\omega}$, that is, $\omega\left(x, H_{v}\right) \neq 0$. Since $u_{n}(z) \geq 0$ in $F_{n}$ and $z \frac{1}{2}$ on $\gamma_{n}(H)=H_{n} \Gamma_{n}$, we have, by Lemma 2.1 , on $H_{v}^{(x)}=H_{v} \cap F_{n}$,

$$
\begin{gathered}
u_{n}(x) \geqq \frac{1}{2} \omega_{n}\left(z, H_{\nu}^{(x)}\right) . \\
\text { Hence, for } n \rightarrow \infty \text {, we have } \\
u(z) \geqq \frac{1}{2} \omega\left(z, H_{\nu}\right) \neq 0 .
\end{gathered}
$$

Thus $u(z) \neq 0$ and hence $u(z)$ is non-constant Dirichlet-Iinite solution of $(A)$ on $\vec{F}$, that is,

$F \notin O_{D}$

Conversely, 11 we assume $F \notin O_{D}$, then it happens only two cases: elther l) there exists at least two linearly independent solutions of (A), being non-constant and Dirichletilnite on $F$, or 2) such a functional space is one dimensional.

If the case 1) happens, then we shall treat the problem in the following way. Let $u_{1}(z)$ and $u_{2}(z)$ be two linearly independent solutions of $(A)$, being non-constant and Dirichlet-finite on $F$, then there are $a$ number $m$ and a point $z_{0} \in F$, such that $u_{1}\left(z_{0}\right)=u_{2}\left(z_{0}\right)$ and

$u_{1}(x) \neq m u_{2}(x)$. Let $U(z)$

$=u_{1}(z)-m u_{2}(z)$, then $U(z)$ is

a non-constant Dirichlet-finfte solution of (A), being $U\left(x_{0}\right)=0$. Without loss of generality, we may assume that $U(z)>0$ for a point $z \in F$. Then, as the desired non-compact connected subregion $G$ of $F$, we can take a connected component of a point-set on which

$U(x)>U\left(x_{0}\right)=0$. Then

$$
D_{G}(U(z)) \leqq D_{F}(U(z))
$$

and $U(z) \equiv 0$ on $C$ (boundary

of $G$ ).

suppose that $G+C=\bar{G}$ is compact, then we can find such a domain $G_{1}$ that $\bar{G}_{1}$ is compact and $G_{1} \supset \bar{G}$ and that $C$ does not touch the boundary $C_{1}$ of $G_{1}$. Obviously $U(x) \in t^{2}\left(G_{1}\right)$, and ther $\theta$ exists the reproducing kernel function $K_{G_{1}}(z, x)$ def'ined in Detinition 2.3 on $G_{1}$. Thus we have

$$
\begin{aligned}
U\left(z_{1}\right) & =D_{G_{1}}\left(U(z), K_{G_{1}}\left(z, z_{1}\right)\right) \\
& \leqq D_{G_{1}}(U(z)) K_{G_{1}}\left(z_{1}, z_{1}\right) .
\end{aligned}
$$

Since $K_{G_{1}}\left(x_{1}, x_{1}\right)$ is unitormly bounc $\theta$ dor $z_{1} \in G, U\left(z_{1}\right)$ is a finite solution of (A) on $G$, being $u(z) \equiv 0$ on $C$. This implies that $U(z)=0$ on $G$, which is absurd. Thus $G$ is: non-compact connected subregion of $E$ and $U(z)$ is the desired solution of (A) on G.

In the case 2), we have

$$
K_{F}(x, \zeta)=\varphi(z) \varphi(\zeta),
$$

where $\varphi(z) \in L^{2}(F), D_{F}(\varphi)=1$ and is non-constant. Suppose that $K_{F}\left(x, \zeta_{1}\right) \equiv m K_{F}\left(z, \zeta_{2}\right)$ lor any $z \in F$ holds, even if we choose two points $\zeta_{1}$ and $\zeta_{2}(\epsilon F)$ whatsoever, where $m$ is a constant, then we have

$$
\varphi\left(\zeta_{1}\right)=m \varphi\left(\zeta_{2}\right) \text {. }
$$

Since $\zeta_{1}$ and $\zeta_{2}$ are arbitrary, we have $m= \pm 1$, but $m \pm-1$ does not arise for $K_{F}(x, \xi) \geq 0$ Thus $m=1$, and hence $\varphi(x) \equiv$ constant, which is absurd. Thus there are two points $\zeta_{1}$ and $\zeta_{2}$ such that

and

$$
K_{F}\left(z, \zeta_{1}\right) \neq m K_{F}\left(z, \zeta_{2}\right)
$$

$$
K_{F}\left(z_{0}, \zeta_{1}\right)=m K_{F}\left(z_{0}, \zeta_{2}\right)
$$

for a suitable point $z_{0}$ on $\vec{F}$. Thus, if we put

$$
U(z)=K_{F^{-}}\left(z, \zeta_{1}\right)-m K_{F}\left(x, \zeta_{2}\right),
$$

and $1 f^{\prime}$ we choose a non-compact connected subregion $G$ of $F$ which is a connected component or a pointset satisfying $U(z)>0$, then we can conclude that $G$ and $U(2)$ are 
the desired domain and function as in the case 1 ). $q \cdot \theta \cdot \alpha$.

§6. $O_{B}$ and $O_{D}$.

Theorem 6.I. If $D_{F}(1,1)=\iint_{F} P d x d y<\infty$ and $\frac{F \in O_{K}}{F \in}$, then $F \in O_{\Omega}$.

Proof. Obviously we have

$$
\begin{aligned}
& \Omega_{m}\left(z, F_{m}\right)=\Omega_{m}\left(z, \Gamma_{m}, F_{m}\right) \\
&=-\frac{1}{2 \pi} \int_{\Gamma_{m}} \frac{\partial}{\partial v} g_{m}(\zeta, z) d s_{\zeta} \\
& K_{m}(\zeta, z)=\frac{1}{2 \pi}\left(N_{m}(\zeta, z)-g_{m}(\zeta, z)\right) \\
& \frac{\partial}{\partial \nu} N_{m}(\zeta, z) \equiv 0 \text { on } \Gamma_{m} .
\end{aligned}
$$

Hence we get

$$
\begin{aligned}
\Omega_{m}\left(z, F_{m}\right) & =\int_{r_{m}} \frac{\partial}{\partial v} K_{m}(\zeta, z) d s_{\zeta} \\
& =D_{F_{m}}\left(1, K_{m}(\zeta, z)\right)_{\zeta} .
\end{aligned}
$$

Thus, by the Schwarz's inequality, we have

$$
0 \leqq \Omega_{m}\left(z, F_{m}\right) \leqq\left(K_{m}(z, z)\right)^{1 / 2}\left(D_{F_{m}}(1,1)\right)^{1 / 2} \text {. }
$$

By the assumption of theorer, right hand side vanish when $m$ tends to

$$
\infty \text {. Thus, } F \in O \Omega \text {. q.e.d. }
$$

Theorem 6.3. If $F \in O_{B}$, then FEOD

Proof. Suppose that $F \in O_{B}$ and $F \in O_{D}$, then, by Theorem 5.1, there exists a pair $(G, U(z))$ such that $G$ is non-compact connected analytic subregion of $\bar{F}$ and $u(z)$ is a non-constant Dirichet-finite solution of (A) and $u(z)=0$ on

$C$. Then on $G$ tnere exists a non-constant bounded Dirichlet-finite solution $v(z)$ of $(A)$ being $v(z)=0$ on $C$ by IJerma 5.4. Therefore, by Theorem 4.1, G $\$ S O \omega$ and hence, by Theorem 4.2, F\& $O_{B}$, wrich' is absurd. $q . e . d$.

Alternative proof of Theorem 6.2.

$$
\text { Suppose that } F \notin O_{D} \text {, then }
$$
there exists a non-constant Dirichletfinite solution $u$ of (A) on $F_{n}$. Without loss of generality wo may assume that the point-set on which $u>0$ is not empty, since we may consider the function $-u$ if necessary. Let $W$ be a compact subregion of $F$, on which $u>0$,

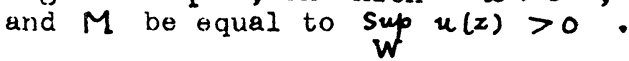
We construct a continuous piecewise continuously diferentiable function $f$ as roliows:

$$
\begin{aligned}
f & =M & & \text { if } z \in W_{1} \text { on which } u>M, \\
& =0 & & \text { if } z \in W_{2} \text { on which } u<0, \\
& =u & & \text { if } z \in W_{3} \text { on which } 0 \leqq u \leqq M .
\end{aligned}
$$

Then we have $D_{F}(f) \leqq D_{F}(u)<\infty$, and $D_{F}(f, u) \gtreqless 0$, since

$$
\begin{aligned}
\infty>D_{w_{1}}(f, u) & =M \iint_{w_{1}} P u d x d y ; \\
D_{w_{2}}(f, u) & =0 ;
\end{aligned}
$$

and

$$
\begin{aligned}
\infty>D_{w_{3}}(f, u) & =D_{w_{3}}(u, u) \\
& \geqq D_{w}(u, u) \supsetneqq 0 .
\end{aligned}
$$

Let $U_{n}$ be a continuous function such that $U_{n} \equiv f$ on $F-F_{n}$ and $U_{n}$ is a solution of (A) on $F_{n}$, then $\left\{U_{n}\right\}$ is a uniformly bounded' sequence, and hence we can select a subsequence of $\left\{U_{n}\right\}$ such that $\lim _{v \rightarrow \infty} U_{n_{v}}=U$ exists and $U$ is a bounded solution of $(A)$. For simplicity's sake we shall retain the original suifices. By Lemma 5.1, we have ${ }_{D_{F}}\left(\stackrel{\text { Or }}{U}_{m}\right) \leqq D_{F}^{m}\left(U_{n}\right) \leqq D_{F}(f)<\infty$. Thus, $\lim _{n \rightarrow \infty} D_{F}\left(U_{n}\right)$ exists and is of Iinite value $D_{F}(U)$. on the other hand, $D_{F}\left(U_{m}, U_{m}-U_{n}\right)=0$ for $m>n$, thus

$$
D_{F}\left(U_{m}-U_{n}\right)=D_{F}\left(U_{n}, U_{n}\right)-D_{F}\left(U_{m}, U_{m}\right) \text {, }
$$

from which we may conclude that $U_{n}$ converges in a stronger sense. Since $D(u)<\infty$, we have the so-called weakly convergent property:

$$
\lim _{n \rightarrow \infty} D_{F}\left(U_{n}, u\right)=D_{F}(U, u)<\infty \text {. }
$$

on the other hand, we have

$$
\begin{aligned}
D_{F}\left(U_{n}, u\right)=D_{F_{n}}\left(U_{n}, u\right)+D_{F-F_{n}}(f, u) \\
=D_{F_{n}}(f, u)+D_{F-F_{n}}(f, u) \\
=D_{F}(f, u) \neq 0 .
\end{aligned}
$$

And hence we have

$$
D_{F}(U, u) \neq 0 \text {. }
$$

Thus $U$ is not identically zero. Thus we obtain the desired result: 


\section{$F \notin O_{D} \quad$ implies $F \notin O_{B}$. \\ $q \cdot \theta \cdot d \cdot$}

Remurk 1. We can arrange our results in the following schema:

$$
O_{G} C O_{G}^{(h)}=O_{\omega}^{(h)} C O_{\omega}=O_{\Omega}=O_{B} C O_{D}=O_{K} \text {. }
$$

2. For any admissible $P(x)$ we obtain the above schema, but it seems to us that we need a special cholce of $P(z)$ in order to bring more precise results. If there exists a single-valued Dirichlet-linite non-constant harmonic function $h(x)$ on $F$, then we have $O_{0}=O_{B}\left(\frac{\partial h}{1 f}\right)^{2}$ we choose as $\left(\frac{\partial h}{\partial x}\right)^{2}+\left(\frac{\partial h}{\partial y}\right)^{2}$ proof is easily performed by considering the fact:

$$
\iint_{F}\left(\left(\frac{\partial h}{\partial x}\right)^{2}+\left(\frac{\partial h}{\partial y}\right)^{2}\right) d x d y<\infty \text { is oqui- }
$$
valent to $D_{F}(1,1)=\iint_{F} P d x d y<\infty$. In this view-point we may say that
a pair $(F, P)$ consisting of a given Riemann surface $F$ and an admissible function $P(z)$ has been classifled, and thus we may write $O_{T}(E)$ where $T$ is $G$ or $w$ or $K$.

3. For a non-linear partial differential equation of elliptic type we shall discuss the results elsewhere. In this case the arguments and results must be modifled.

$$
\text { 4. If } P_{1}(z), P_{2}(z)\left(P_{1}(z) \leqq P_{2}(z)\right)
$$
are two admissible 'runctions in our original case, then we have the following results:

$$
\begin{aligned}
& \text { 1) } O_{G}\left(P_{1}\right) \supset O_{G}\left(P_{2}\right) \text {, } \\
& \text { 11) } O_{\omega}\left(P_{1}\right) \subset O_{\omega}\left(P_{2}\right) \text {, } \\
& \text { 111) } O_{K}\left(P_{1}\right) \subset O_{K}\left(P_{2}\right) \text {. }
\end{aligned}
$$

These results were obtained al ready by Bergman-Schiffer [2] wi thout considerations of null-boundary. They considered only the case of planar schlicht compact analytic domains, but their proof's remain valid also in case of Riemann surfaces.

\footnotetext{
5. In a schlicht domain we may take $P(z)$ without the contormally covariant property: $P(z)=P\left(z^{\prime}\right)\left|\frac{d z^{\prime}}{d z}\right|^{2}$ for example, constant $k(\neq 0)$. In these cases our arguments remain valld with some exceptions, but our main results, that is,
}

$$
O_{G} C O_{\omega}=O_{\Omega}=O_{B} C O_{D}=O_{K}
$$

also remain valid. Moreover we can define other several Hilbert spaces with the various different metrics, and we can deline other several corresponding kernel functions and hence other sorts of null-set. Cf. M. Schiffer [1], Bergman-Schiffer [2].

\section{\$7. Riemann Surlaces of Finfte Genus.}

Lemma 7.1. Let $E$ be a compact set on $F_{i}$, being compact subregion of $F$, and $\varphi(x)$ be an arbitrary bounded solution of (A) in $F_{1}-E$ - A necessary and sutilcient condition in order that $\varphi(z)$ is prolongable on $E$ in the sense of (A) is that $\omega\left(z, E, F_{1}\right)=0$.

Proof. Sufficiency. Let $\Gamma$ be a Iinite number of Jordan curves, surrounding $E$, and belonging to $F_{1}$ with boundary $C$, and $F_{C}$ be $F_{1}-F_{\Gamma}$, where $F_{\Gamma}$ is the domain bounded by $\Gamma$. Then $\omega\left(z, E, F_{1}\right)$ $\equiv 0$ means that $\omega\left(z, \Gamma, F_{c}\right)<\varepsilon$ for any $\Gamma$ sufficientiy near to $E$. Let $\varphi_{0}(z)$ be a solution of $(A)$ in $F_{1}$, coinciding with

$\varphi(z)$ on $C$. Ir we introduce the function $\Omega\left(z, C, F_{1}\right)$, then we have, from Lemma 2.I, $\left|\varphi_{0}(z)\right|$ $\leqq M \Omega\left(z, C, F_{1}\right)$, where $M=\sup _{F_{1}}|\varphi(z)|$.

$$
\text { Let } \Phi(z)=\varphi(z)-\varphi_{0}(z) \text {, then }|\Phi(z)|
$$

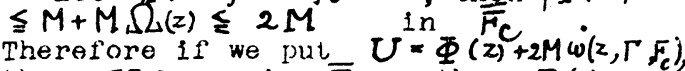
then $U \geq 0$ in $\overline{F_{C}}$; thus $\Phi(x)$ $z-2 M \omega(z) z-2 M \&$ and hence $\Phi(z) \geqq 0$ In $F_{1}-E$.

Similarly if we consiaer the tunction $V=\Phi-2 M \omega$, then $\Phi(z)$

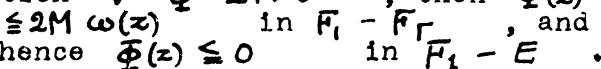

Thereiore $\Phi(z)=0$ in $\bar{F}_{1}-E$. Thus, if we define $\varphi(z)=\varphi_{0}(z)$ in $\vec{E}$ and $\varphi(z)=\varphi(z)$ in $\bar{F}-\bar{E}$, then $\varphi(z)$ is bounded and satis fles $\Delta \varphi=E \varphi$ in $\bar{E}$.

Necessity. There is at least a point $z$ in $F_{1}-E$, such that $\omega\left(z, E, F_{1}-E\right)>0$. On the other hand, $\omega\left(Z, E, F_{1}-E\right)$ is bounded in $F_{1}-E$, and therefore, by the assumption, $\omega\left(z, E, F_{1}-E\right)$ must be prolongable on $E$ and of finite value. On the other hand, $\omega \equiv 0$ on $C$. Since $F_{1}$ is a compact subregion of $\bar{H}, \quad \omega \equiv 0$ on q. $\bar{F}_{1}$. . which contradicts $\omega>0$

The following theorem yields another sul'ticlency proot of Theorem 3.3 in the case of Riemann suriace of I'inite genus. 
Theorem 7.1. Iset $F$ be a liemann surfece of l'inite gerius, and $E$ a compact subset of $F$. When $\bar{F}-E \in O \omega$ implies $F-E \in O_{B}$ and vice versa.

Proof. If $F-E \in O \omega$ and $i t^{\prime}$ $u(z)$ is an arbitrary bounded nonconstant solution of (A) on the neighbourhood $F_{1}$ of $E$, then from Theorem 7.1 we $s \in \theta$ that' $\mu(z)$ is prolongable on $E$ with regard to (A) and is of finite value on $E$. on the other hand, $\Omega(z, E)$ $=\lim _{n \rightarrow \infty} \Omega\left(z, \Gamma_{n}, F_{n}\right)$ is a bounded solution of $f^{(A)}$ on $\bar{F}_{1}$, therefore $\Omega(x, E)$ is prolongable on $E$ and is of finite value on $E$. Thus $\Omega(z, E)$ is a I'ilite non-negative solution of (A) on the whole $E$, and hence from the subharmonicity of $\Omega(z, E)$, it reduces to a constant, that is, zero. This shows that $F-E \in O_{B}$.

The converse is immediately obtained by the necessity parts of Theorem 3.3 and Theorem 3.1. $q \cdot e \cdot d$.

(*) Recelved June 26, 1952 。

N.Aronszajn. [l] : Theory of reproducing kernels, Trans. Amer. Math. Soc. 68(1950) 337-404.

S.Bergman. [1] : Kernel function and conformal mapping. Math. Survey. (1951).

S.Bertrman-M.Schiffer. [I] : A representation of Green's and Neumann's functions in the theory of partial differential equations of second order. Duke Math. Journ. 14 (1947) 609-638.

[2] : Kernel ounctions in the theory of partial differential equations of elifptic type. Duke Math. Journ. $15(1948) 535-566$.

R.Courant-1.Hilbert. [1]: Methoden der nathematischen Physik. Bd.II (1937)。

D.Hilbert. [I] : Grundzüge einer aligemeinen Theorie der linearen Integralgleichungen. Leipzig. (1912). E.Holmgren. [I] : Über die Existenz der Grundlösung bei einer linearen pertielien Differentialgleichung der
2. Ordnung von elliptischem Typus. Math. Ann. 58(1904) 404-412.

L.Lichtenstein. [1] : Randwertaufgaben der Theorle der linearen partielien Diliterential ordnung vom elliptischen Typus. I. Crelles Journ. 142(1913) 1-40.

[2]: Randwertaulgaben der Theorie der linearen partielien Differentialfleichung zweiter oranung vom elliptischen Typus. II. Crelles Journ. 143 (i913) $51-105$.

A.Mor1. [1] : On the existence of harmonic lunctions on a Riemann surface. Scl. Rep. Tokyo Univ.6(1951) 247-257.

C.Neumann. [l] : Vorlesungen über Riemann's Theorle der Abel'schen Integrale。 Leipzig ( 1884).

R.Nevanlinna。 [1] : Über das alternierende Verfahren von Schwarz. Crelles Journ. 180)(1939) 121-128。

H.L.Royden. [I] : Some remarks on open Riemann surfices. Ann. Acad. Sci. Fenn. AI. No.85(i951).

E.Plcard. [I]: Sur line classe d'équations linéaires aux dérivées partielles du second ordre. Acta Math. 12(1889). 323-338。

[2] : Memolre sur la théorie des équations aux dérivées partielles et Ia méthode des approximations successives. Journ. Math. Pures et Appl. VI. (1890) 145-210.

M.Schiffer。[1] : Various types of orthogonalization. Duke Math. Journ. 17(1950) 329-366.

K.I.Virtanen. [I] : Über die Existenz von beschränkten harmonischen Funktionen auf of'enen Riemannschen Flächen. Ann. hcad. Sci. Fenn. AI. No.75 (1950)。

Departnient of Mathematics, Tokyo Institute of Technology. 